Internat. J. Math. \& Math. Sci.

Vol. 24, No. 4 (2000) 265-276

S0161171200001605

(c) Hindawi Publishing Corp.

\title{
WAVES DUE TO INITIAL DISTURBANCES AT THE INERTIAL SURFACE IN A STRATIFIED FLUID OF FINITE DEPTH
}

\author{
PRITY GHOSH, UMA BASU, and B. N. MANDAL
}

(Received 12 December 1996 and in revised form 6 October 1997)

\begin{abstract}
This paper is concerned with a Cauchy-Poisson problem in a weakly stratified ocean of uniform finite depth bounded above by an inertial surface (IS). The inertial surface is composed of a thin but uniform distribution of noninteracting materials. The techniques of Laplace transform in time and either Green's integral theorem or Fourier transform have been utilized in the mathematical analysis to obtain the form of the inertial surface in terms of an integral. The asymptotic behaviour of the inertial surface is obtained for large time and distance and displayed graphically. The effect of stratification is discussed.
\end{abstract}

Keywords and phrases. Stratified fluid, Boussinesq approximation, initial disturbance, inertial surface.

2000 Mathematics Subject Classification. Primary 76B70.

1. Introduction. The classical two-dimensional problem of generation of unsteady motion in deep water due to initial surface disturbances in the form of initial elevation or impulse concentrated at a point on the free surface was studied in the treatise of Lamb [4] and Stoker [9] assuming linear theory. Fourier transform technique was used in the mathematical analysis and the free surface elevation was obtained in the form of an infinite integral which was then evaluated asymptotically for large time and distance by the method of stationary phase. Kranzer and Keller [3] considered the three-dimensional unsteady motion in water of uniform finite depth due to initial surface impulse or elevation applied on a circular area on the free surface. Chaudhuri [1] and Wen [10] extended these results for surface impulse and elevation across arbitrary regions.

When an ocean is covered by an inertial surface consisting of a thin but uniform distribution of non-interacting materials such as broken ice, a number of problems of unsteady motion created due to initial disturbances at the inertial surface were considered by Mandal [5], Mandal and Ghosh [6, 7], Mandal and Mukherjee [8]. Study of these classes of problems involving inertial surface has acquired some importance because of increase in the various types of scientific activities in antartica in the vicinity of which the ocean is sometimes covered by broken ice.

In all the above studies, the ocean is assumed to be a homogeneous fluid. However, because of salinity the density of the ocean increases with depth and it is thus realistic if one models an ocean as a stratified fluid.

For a weakly stratified fluid with constant Brunt-Vaisala parameter, Debnath and Guha [2] formulated the problem of wave generation due to prescribed initial disturbance of the free surface in terms of an acceleration potential and obtained 
the free surface profile asymptotically for large time and distance far away from the region of disturbance. However, their modelling a deep ocean by a stratified fluid of infinite depth with constant Brunt-Vaisala parameter is questionable since the density at very large depth becomes very large even if the Brunt-Vaisala parameter is small. In the present paper, we study the problem of wave generation due to initial disturbances in a weakly stratified fluid of finite depth covered by an inertial surface. The initial disturbances are prescribed at the inertial surface. Assuming linear theory the problem is formulated in terms of pressure under Boussinesq approximations and constant but small Brunt-Vaisala parameter. By using Laplace transform technique, the initial value problem is reduced to a boundary value problem which is then solved by two methods, one based on an appropriate use of Green's integral theorem and the other on Fourier transform. The form of the inertial surface is then obtained in terms of an integral. This integral is evaluated asymptotically for large times and distances by the method of stationary phase when the initial disturbances at the inertial surface is concentrated at a point taken as the origin. The asymptotic form of the inertial surface profile is depicted graphically and compared with the result for an ideal fluid covered by an inertial surface. It is found that the effect of weak stratification is not of much significance.

2. Formulation of the problem. We consider an incompressible inviscid weakly stratified fluid of uniform finite depth $h$ covered by an inertial surface composed of a thin but uniform distribution of disconnected floating materials of area density $\rho_{0}(0) \epsilon(\epsilon \geq 0)$, where $\rho_{0}(0)$ is the density of the fluid at the top. It may be noted that $\epsilon=0$ corresponds to a fluid with a free surface. We choose a rectangular cartesian coordinate system in which the $y$-axis is taken vertically downwards into the fluid, $y=0$ is the position of the inertial surface at rest. The motion in the fluid is generated due to an initial disturbance prescribed on the inertial surface in the form of an initial depression of the inertial surface or initial impulse. We assume the resulting motion to be two-dimensional. We denote by $p(x, y, t)$ and $\rho(x, y, t)$ the perturbed pressure and density of the fluid, respectively, while $\rho_{0}(y)$ denotes the density of the fluid at rest, $u(x, y, t)$ and $v(x, y, t)$ are respectively, the horizontal and vertical components of velocity.

Under the assumption of linear theory the relevant equations satisfied in the fluid region are

$$
u_{x}+v_{y}=0, \quad \rho_{t}+v \rho_{0}^{\prime}(y)=0, \quad \rho_{0} u_{t}=-p_{x}, \quad \rho_{0} v_{t}=-p_{y}+g \rho .
$$

Combining the kinematic and dynamic conditions at the inertial surface, we find

$$
p-\epsilon p_{y}=m g+\Pi-\epsilon g \rho-g \rho_{0} \eta \quad \text { on } y=0, t>0,
$$

where $\eta(x, t)$ is the depression of the inertial surface, $g$ is the gravity and $\Pi$ is the atmospheric pressure. The initial conditions are

$$
u=v=0, \quad \text { at } t=0 \quad \eta(x, t)=\eta_{0}(x) \quad \text { at } t=0,
$$

where $\eta_{0}(x)$ is the prescribed initial depression of the inertial surface. 
Let $\bar{p}(x, y ; s), \bar{\eta}(x ; s), \bar{\rho}(x, y ; s)$, and $\bar{v}(x, y ; s)$ denote the Laplace transform of $p(x, y, t), \eta(x, t), \rho(x, y, t)$, and $v(x, y, t)$ in time, respectively.

Under Boussinesq approximation, equations (2.1) produce

$$
\bar{p}_{x x}+\lambda^{2} \bar{p} y y=0, \quad 0 \leq y \leq h,-\infty<x<\infty,
$$

where $\lambda=s /\left(s^{2}+N^{2}\right)^{1 / 2}$ is chosen such that $\operatorname{Re} \lambda>0$, and

$$
N^{2}=\frac{g}{\rho_{0}} \rho_{0}^{\prime}(y)
$$

is the Brunt-Vaisala parameter which is assumed to be constant and small. The smallness of $N$ characterizes weakly stratified fluid.

We now solve (2.4) along with the boundary conditions

$$
\begin{gathered}
\frac{s^{2}}{\lambda^{2}} \bar{p}(x, 0 ; s)-\left(g+\epsilon s^{2}\right) \bar{p}_{y}=-\frac{s g \rho_{0}(0) \eta_{0}(x)}{\lambda^{2}} \text { on } y=0, \\
\bar{p}_{y}=0 \quad \text { on } y=h .
\end{gathered}
$$

$\bar{p}(x, y ; s)$ is obtained in the next section by employing two methods, one based on an appropriate use of Green's integral theorem and the other on Fourier integral transform.

\section{Methods of solution}

3.1. Method based on Green's integral theorem. We use the transformation $x=X$, $y=\lambda Y$ and denote $\bar{P}(X, Y)=\bar{p}(X, \lambda Y)$, then $\bar{P}(X, Y)$ satisfies

$$
\nabla^{2} \bar{P}=0 \quad \text { in } 0 \leq Y \leq \frac{h}{\lambda},-\infty<X<\infty,
$$

with

$$
\begin{gathered}
\frac{s^{2}}{\lambda} \bar{P}(X, 0 ; s)-\left(g+\epsilon s^{2}\right) \bar{P}_{Y}=-\frac{s g \rho_{0}(0) \eta_{0}(X)}{\lambda} \text { on } Y=0, \\
\bar{P}_{Y}=0 \quad \text { on } Y=\frac{h}{\lambda}=h^{\prime} .
\end{gathered}
$$

The boundary value problem described by (3.1) and (3.2) is now solved by an appropriate use of Green's integral theorem to the functions $G\left(X, Y ; X^{\prime}, Y^{\prime} ; s\right)$ and $\bar{P}(X, Y)$, where $G$ satisfies

$$
\begin{aligned}
& \nabla^{2} G=0, \quad 0 \leq Y \leq h^{\prime} \text { except at }\left(X^{\prime}, Y^{\prime}\right), \\
& \frac{s^{2}}{\lambda} G-\left(g+\epsilon s^{2}\right) G_{Y}=0 \quad \text { on } Y=0, \\
& G \longrightarrow \ln R \quad \text { as } R=\left[\left(X-X^{\prime}\right)^{2}+\left(Y-Y^{\prime}\right)^{2}\right]^{1 / 2} \longrightarrow 0, \\
& G_{Y}=0 \quad \text { on } Y=h^{\prime} \\
& G \longrightarrow 0 \quad \text { as } R \rightarrow \infty .
\end{aligned}
$$


Then, $G\left(X, Y ; X^{\prime}, Y^{\prime} ; s\right)$ can be obtained as

$$
\begin{aligned}
& G\left(X, Y ; X^{\prime}, Y^{\prime} ; s\right) \\
& \quad=U\left(X, Y ; X^{\prime}, Y^{\prime}\right)-2 \int_{0}^{\infty} \frac{\cosh k\left(h^{\prime}-Y^{\prime}\right) \cosh k\left(h^{\prime}-Y\right)}{D(k)\left(s^{2}+\lambda \mu^{2}\right)} \frac{\lambda \mu^{2}}{k \sinh k h^{\prime}} \cos k\left(X-X^{\prime}\right) d k,
\end{aligned}
$$

where

$$
\begin{aligned}
U\left(X, Y ; X^{\prime}, Y^{\prime}\right)=\ln \frac{R}{R^{\prime}}-2 \lambda \int_{0}^{\infty}[ & \frac{\cosh k\left(h^{\prime}-Y^{\prime}\right) \cosh k\left(h^{\prime}-Y\right)}{D(k)} \\
& \left.+\frac{\exp \left(-k h^{\prime}\right) \sinh k Y^{\prime} \sinh k Y}{k}\right] \frac{\cos k\left(X-X^{\prime}\right)}{\cosh k h^{\prime}} d k
\end{aligned}
$$

with

$$
\begin{gathered}
R^{\prime}=\left[\left(X-X^{\prime}\right)^{2}+\left(Y+Y^{\prime}\right)^{2}\right]^{1 / 2}, \\
D(k)=\cosh k h^{\prime}+\epsilon k \lambda \sinh k h^{\prime}, \quad \mu^{2}=\frac{g k \sinh k h^{\prime}}{D(k)} .
\end{gathered}
$$

Thus we find

$$
\bar{P}\left(X^{\prime}, Y^{\prime}\right)=\frac{g \rho_{0}(0) s}{\pi\left(g+\epsilon s^{2}\right) \lambda} \int_{-\infty}^{\infty} G\left(X, 0 ; X^{\prime}, Y^{\prime} ; s\right) \eta_{0}(X) d X .
$$

Using (2.4) and reverting to the original variables we find that

$$
\begin{aligned}
\bar{p}(x, y) & \equiv \bar{P}\left(x, \frac{y}{\lambda}\right) \\
& =-\frac{g \rho_{0}(0) s}{\Pi} \int_{0}^{\infty} \frac{\cosh k\left(h^{\prime}-y / \lambda\right)}{D(k)\left(s^{2}+\lambda \mu^{2}\right)} \int_{-\infty}^{\infty} \eta_{0}\left(X^{\prime}\right) \cos k\left(x-X^{\prime}\right) d X^{\prime} d k .
\end{aligned}
$$

3.2. Method based on Fourier transform technique. Employing Fourier transform in $x$, the boundary value problem described by (2.4) and (2.6) reduces to

$$
\begin{aligned}
& \overline{\bar{p}}^{\prime \prime}(y)-\frac{\xi^{2}}{\lambda^{2}} \overline{\bar{p}}=0, \quad 0 \leq y \leq h, \\
& \frac{s^{2}}{\lambda^{2}} \overline{\bar{p}}-\left(g+\epsilon s^{2}\right) \overline{\bar{p}}^{\prime}(y)=-\frac{s g \rho_{0}(0) \bar{\eta}_{0}(\xi)}{\lambda^{2}} \text { on } y=0, \\
& \overline{\bar{p}}^{\prime}(y)=0 \quad \text { on } y=h,
\end{aligned}
$$

where $\overline{\bar{p}} \equiv \overline{\bar{p}}(\xi, y ; s)$ and $\bar{\eta}_{0}(\xi)$ are the Fourier transform of $p(x, y ; s)$ and $\eta_{0}(x)$, respectively. Equation (3.10) is an ordinary differential equation and its solution satisfying (3.11) and (3.12) is given by

$$
\overline{\bar{p}}=-s g \rho_{0}(0) f(\xi ; y) \bar{\eta}_{0}(\xi),
$$


where

$$
f(\xi ; y)=\frac{\cosh (\xi / \lambda)(h-y)}{D(\xi)\left(s^{2}+\lambda \mu^{2}(\xi)\right)},
$$

so that $f(\xi ; y)$ is an even function of $\xi$. Using Fourier inversion, we find $\bar{p}(x, y)$, which is the same as given by (3.9).

Now $\bar{\eta}(x ; s)$ can be expressed in terms of $\bar{p}(x, y ; s)$ given by

$$
\bar{\eta}(x ; s)=\frac{1}{g \rho_{0}}\left[\epsilon \lambda^{2} \frac{\partial \bar{p}}{\partial y}(x, 0 ; s)-\bar{p}(x, 0 ; s)\right]
$$

so that, after using (3.9), we find

$$
\bar{\eta}(x ; s)=\frac{1}{\pi} \int_{0}^{\infty} \frac{s}{s^{2}+\lambda \mu^{2}} \int_{-\infty}^{\infty} \eta_{0}\left(X^{\prime}\right) \cos k\left(x-X^{\prime}\right) d X^{\prime} d k .
$$

By Laplace inversion, equation (3.16) will produce $\eta(x, t)$ in principle.

Now let us choose the initial displacement of the inertial surface to be concentrated at the origin, then $\eta_{0}\left(X^{\prime}\right)$ is taken as $l^{2} \delta(x)$, where $l$ is a typical length, then

$$
\bar{\eta}(x ; s)=\frac{l^{2}}{\pi} \int_{0}^{\infty} \frac{s}{s^{2}+\lambda \mu^{2}} \cos k x d k
$$

which, when written fully, is equivalent to

$$
\bar{\eta}(x ; s)=\frac{l^{2}}{\pi} \int_{0}^{\infty} \frac{s\left(1+N^{2} / s^{2}\right)^{1 / 2}+\epsilon k s \tanh \left[k h\left(1+N^{2} / s^{2}\right)^{1 / 2}\right]}{s^{2}\left(1+N^{2} / s^{2}\right)^{1 / 2}+\left(\epsilon k s^{2}+g k\right) \tanh \left[k h\left(1+N^{2} / s^{2}\right)^{1 / 2}\right]} \cos k x d k .
$$

For the purpose of Laplace inversion, we note that $s= \pm i N$ are not branch points. This can be ascertained by noting that an even function of $\left(1+N^{2} / s^{2}\right)^{1 / 2}$ results after expanding the hyperbolic functions. Thus the only contribution to the Laplace inversion integral comes from the zeros of the denominator in the complex $s$-plane. The denominator has no real zero, and the only zeros are purely imaginary given by

$$
s= \pm i w(k)
$$

where $w(k)>N$ and

$$
w^{2}(k)=\frac{2 g k \tanh k h+N^{2}(1-2 k h / \sinh 2 k h)}{2(1+\epsilon k \tanh k h)}+O\left(N^{4}\right) .
$$

Thus,

$$
\eta(x, t)=\frac{2 l^{2}}{\pi} \int_{0}^{\infty} \frac{F(k)}{G(k)} \cos w(k) t \cos k x d k
$$

where

$$
\begin{aligned}
F(k)= & \left(w^{2}-N^{2}\right)+\epsilon k w\left(w^{2}-N^{2}\right)^{1 / 2} \tanh \left[\frac{k h\left(w^{2}-N^{2}\right)^{1 / 2}}{w}\right] \\
G(k)= & \left(2 w^{2}-N^{2}\right)+2 \epsilon k w\left(w^{2}-N^{2}\right)^{1 / 2} \tanh \left[\frac{k h\left(w^{2}-N^{2}\right)^{1 / 2}}{w}\right] \\
& +\left(\epsilon k-\frac{g k}{w^{2}}\right) N^{2} k h \operatorname{sech}^{2}\left[\frac{k h\left(w^{2}-N^{2}\right)^{1 / 2}}{w}\right]
\end{aligned}
$$


Thus the depression of the inertial surface is obtained in terms of an infinite integral. We note that in the absence of stratification, $N=0$, and for deep water (3.21) produces

$$
\eta(x, t)=\frac{l^{2}}{\pi} \int_{0}^{\pi} \cos \left[\frac{g k}{1+\epsilon k}\right]^{1 / 2} t \cos k x d k
$$

which coincides with the result obtained by Mandal [5] earlier except for the scaling factor $l^{2}$.

In (3.21) we use the non-dimensional substitutions $\tilde{x}=x / l, \tilde{t}=(g / l)^{1 / 2} t$, to obtain the non-dimensional form of the inertial surface depression as

$$
\tilde{\eta}(\tilde{x}, \tilde{t}) \approx \frac{\eta(x, t)}{l}=\frac{2 l}{\pi} \int_{0}^{\infty} \frac{F(k)}{G(k)} \cos \left[w(k)\left(\frac{l}{g}\right)^{1 / 2} \tilde{t}\right] \cos (l k \tilde{x}) d k .
$$

In the next section, asymptotic form of $\tilde{\eta}(\tilde{x}, \tilde{t})$ will be obtained for large $\tilde{x}$ and large $\tilde{t}$ such that $\tilde{x} / \tilde{t}$ remains finite.

4. Asymptotic expansion. To obtain the asymptotic form of $\tilde{\eta}(\tilde{x}, \tilde{t})$, we use the method of stationary phase. Now (3.24) can be written in the equivalent form

$$
\begin{aligned}
\tilde{\eta}(\tilde{x}, \tilde{t})=\frac{l}{2 \pi} \int_{0}^{\infty} \frac{F(k)}{G(k)}\{ & \exp \left[i \tilde{t}\left(w(k)\left(\frac{l}{g}\right)^{1 / 2}+\frac{k l \tilde{x}}{\tilde{t}}\right)\right] \\
& +\exp \left[-i \tilde{t}\left(w(k)\left(\frac{l}{g}\right)^{1 / 2}+\frac{k l \tilde{x}}{\tilde{t}}\right)\right] \\
& +\exp \left[i \tilde{t}\left(w(k)\left(\frac{l}{g}\right)^{1 / 2}-\frac{k l \tilde{x}}{\tilde{t}}\right)\right] \\
& \left.+\exp \left[-i \tilde{t}\left(w(k)\left(\frac{l}{g}\right)^{1 / 2}-\frac{k l \tilde{x}}{\tilde{t}}\right)\right]\right\} d k .
\end{aligned}
$$

Let

$$
\phi(k)=\phi(k ; \tilde{x}, \tilde{t})=w(k)\left(\frac{l}{g}\right)^{1 / 2}-\frac{k l \tilde{x}}{\tilde{t}} .
$$

The first two integrals in (4.1) have no stationary point in the range of integration so that they do not contribute. The third and fourth integrals have stationary points given by

$$
\phi^{\prime}(k)=0 .
$$

Now

$$
\phi^{\prime}(0)=l\left\{\left[\left(\frac{h}{l}\right)^{1 / 2}\left(1+\frac{N^{2} h}{3 g}\right)^{1 / 2}\right]-\frac{\tilde{x}}{\tilde{t}}\right\} \equiv l\left(M-\frac{\tilde{x}}{\tilde{t}}\right),
$$

where

$$
M=\left[\left(\frac{h}{l}\right)^{1 / 2}\left(1+\frac{N^{2} h}{3 g}\right)^{1 / 2}\right], \quad \phi^{\prime}(\infty)=-\frac{\tilde{x}}{\tilde{t}}
$$


Also we have verified that $\phi^{\prime \prime}(k)<0$ for $0<k<\infty$ so that $\phi^{\prime}(k)$ is monotone decreasing for $0<k<\infty$. Now for $\tilde{x} / \tilde{t}>M$, both $\phi^{\prime}(0)$ and $\phi^{\prime}(\infty)$ are negative. Hence there is no zero of $\phi^{\prime}(k)$ for $0<k<\infty$ so that there is no stationary point in this case. However, for $\tilde{x} / \tilde{t}<M, \phi^{\prime}(0)$ is positive while $\phi^{\prime}(\infty)$ is negative and since $\phi^{\prime}(k)$ is a monotone decreasing function for $k>0, \phi^{\prime}(k)$ has a unique zero in $(0, \infty)$ so that there exists only one stationary point at $k=k_{0}$, say. Finally when $\tilde{x} / \tilde{t}=M$, $\phi^{\prime}(0)=0$ so that there is a stationary point at $k=0$. But as $\phi^{\prime \prime}(0)=\infty$, it gives a smaller contribution than the $\tilde{x} / \tilde{t}<M$ case, so that its contribution can be neglected. Now applying the method of stationary phase to the third and fourth integrals and combining we find

$$
\tilde{\eta}(\tilde{x}, \tilde{t}) \approx \frac{l}{\pi}\left[\frac{2 \pi l^{3 / 2} g^{1 / 2}}{\tilde{t}\left|\phi^{\prime \prime}\left(k_{0}\right)\right|}\right]^{1 / 2} \frac{F\left(k_{0}\right)}{G\left(k_{0}\right)} \cos \left(\tilde{t} \phi\left(k_{0}\right)-\frac{\pi}{4}\right) .
$$

We may note that in (4.6), $k_{0}$ is a function of $\tilde{x}$ and $\tilde{t}$, and can be evaluated numerically for given $\tilde{x}$ and $\tilde{t}$ for which $\tilde{x} / \tilde{t}<M$ from the transcendental equation

$$
\phi^{\prime}\left(k_{0} ; \tilde{x}, \tilde{t}\right)=0
$$

and thus $\tilde{\eta}(\tilde{x}, \tilde{t})$ can be obtained numerically. In Figures 5.1 and $5.2, \tilde{\eta}(\tilde{x}, \tilde{t})$ is depicted graphically against $\tilde{x}$ (for fixed $\tilde{t}$ ) and $\tilde{t}$ (for fixed $\tilde{x}$ ), respectively, for various values of other parameters. We note that for a homogeneous fluid of infinite depth, equation (4.6) reduces to the classical result

$$
\tilde{\eta}(\tilde{x}, \tilde{t}) \approx \frac{1}{\tilde{x} \pi^{1 / 2}}\left(\frac{\tilde{t}^{2}}{4 \tilde{x}}\right)^{1 / 2} \cos \left(\frac{\tilde{t}^{2}}{4 \tilde{x}}-\frac{\pi}{4}\right) .
$$

When the disturbance is in the form of an impulsive pressure $I(x)$ per unit area applied to the inertial surface, then the condition (2.6) is to be replaced by

$$
\frac{s^{2}}{\lambda} \bar{p}(x, 0 ; s)-\left(g+\epsilon s^{2}\right) \bar{p}_{y}=\frac{s^{2}}{\lambda} I(x) \quad \text { on } y=0
$$

so that in this case, following the same procedure, we obtain instead of (3.9),

$$
\bar{p}(x, y)=\frac{s^{2}}{\pi} \int_{0}^{\infty} \frac{\cosh k\left(h^{\prime}-y / \lambda\right)}{D(k)\left(s^{2}+\lambda \mu^{2}\right)} \int_{-\infty}^{\infty} I\left(x^{\prime}\right) \cos k\left(x-x^{\prime}\right) d x^{\prime} d k .
$$

Thus instead of (3.16), we obtain

$$
\bar{\eta}(x, s)=-\frac{1}{\pi g \rho_{0}(0)} \int_{0}^{\infty} \frac{s^{2}}{s^{2}+\lambda \mu^{2}} \int_{-\infty}^{\infty} I\left(x^{\prime}\right) \cos k\left(x-x^{\prime}\right) d x^{\prime} d k .
$$

If we assume the impulse to be concentrated at the origin, then

$$
I\left(x^{\prime}\right)=A \delta\left(x^{\prime}\right)
$$


where, $A$ is the total impulse per unit length so that

$$
\bar{\eta}(x, s)=-\frac{A}{\pi g \rho(0)} \int_{0}^{\infty} \frac{s^{2}}{s^{2}+\lambda \mu^{2}} \cos k x d k .
$$

Laplace inversion gives

$$
\eta(x, t)=\frac{2 A}{\pi g \rho(0)} \int_{0}^{\infty} \frac{w(k) F(k)}{G(k)} \sin [w(k) t] \cos k x d k .
$$

We again use a similar non-dimensional substitution in (4.14). We get the nondimensional form of the inertial surface depression as

$$
\bar{\eta}(\tilde{x}, \tilde{t})=\frac{2 A}{\pi g \rho_{0}(0) l} \int_{0}^{\infty} \frac{w(k) F(k)}{G(k)} \sin \left[w(k)\left(\frac{l}{g}\right)^{1 / 2} \tilde{t}\right] \cos (l k \tilde{x}) d k .
$$

By the use of stationary phase, the asymptotic form of $\tilde{\eta}(\tilde{x}, \tilde{t})$ is given by

$$
\tilde{\eta}(\tilde{x}, \tilde{t}) \approx \frac{A}{\pi g \rho_{0}(0) l}\left[\frac{2 \pi g^{1 / 2}}{l^{1 / 2} \tilde{t}\left|\phi^{\prime \prime}\left(k_{0}\right)\right|}\right]^{1 / 2} \frac{w\left(k_{0}\right) F\left(k_{0}\right)}{G\left(k_{0}\right)} \sin \left[\tilde{t} \phi\left(k_{0}\right)-\frac{\pi}{4}\right] .
$$

We note that for a homogeneous fluid of infinite depth, (4.15) reduces to the classical result

$$
\tilde{\eta}(\tilde{x}, \tilde{t}) \approx \frac{A}{4(\pi g)^{1 / 2} \rho_{0}(0) l^{5 / 2}} \frac{\tilde{t}^{2}}{\tilde{x}^{5 / 2}} \sin \left(\frac{\tilde{t}^{2}}{4 \tilde{x}}-\frac{\pi}{4}\right),
$$

$\rho_{0}(0)$ being the density of the homogeneous fluid.

5. Discussion. To display the effect of stratification and the inertial surface on the wave motion generated by the initial disturbances, the non-dimensional asymptotic forms of $\tilde{\eta}(\tilde{x}, \tilde{t})$ are depicted graphically against $\tilde{x}$ for fixed $\tilde{t}$ and against $\tilde{t}$ for fixed $\tilde{x}$ such that $\tilde{x} / \tilde{t}<M$. We need to compute $k_{0} h$, where $k_{0}$ is the unique positive root of the transcendental equation $\phi^{\prime}(k)=0$. This root is obviously a function of $\tilde{x}, \tilde{t}$, and other parameters. A representative set of values of $k_{0} h$ for different values of $\tilde{x}$ and $N^{2} h / g$ choosing $\tilde{t}=8, \epsilon / h=0.001, l / h=1$ is given in Table 5.1 while in Table 5.2 for various values of $\tilde{t}$ and $\epsilon / h$, choosing $\tilde{x}=1, N^{2} h / g=0.01, l / h=1$.

TABLE 5.1. $\tilde{t}=8, \epsilon / h=0.001$.

\begin{tabular}{cccccccc}
\hline$N^{2} h / g$ & 0 & 0.01 & 0.1 & 0.5 & 1 & 2 & 3 \\
\hline$\tilde{x}$ & $k_{0} h$ & $k_{0} h$ & $k_{0} h$ & $k_{0} h$ & $k_{0} h$ & $k_{0} h$ & $k_{0} h$ \\
\hline 1.5 & 6.964902 & 6.959943 & 6.915305 & 6.717025 & 6.469552 & 5.977292 & 5.493912 \\
2.5 & 2.750016 & 2.747885 & 2.729317 & 2.658495 & 2.592053 & 2.508299 & 2.462785 \\
3.5 & 1.777404 & 1.777614 & 1.779624 & 1.789625 & 1.803501 & 1.832352 & 1.860293 \\
4.5 & 1.305230 & 1.306351 & 1.316251 & 1.356073 & 1.398425 & 1.466803 & 1.521161 \\
5.5 & 0.972679 & 0.974439 & 0.989769 & 1.049653 & 1.110611 & 1.204210 & 1.275214 \\
\hline
\end{tabular}


TABLE 5.2. $\tilde{x}=1, N^{2} h / g=0.01$.

\begin{tabular}{cccc}
\hline$\epsilon / h$ & 0 & 0.001 & 0.01 \\
\hline$\tilde{t}$ & $k_{0} h$ & $k_{0} h$ & $k_{0} h$ \\
\hline 2.0 & 1.518101 & 1.514281 & 1.481504 \\
4.0 & 4.033437 & 3.988572 & 3.653465 \\
6.0 & 8.995004 & 8.762423 & 7.283130 \\
7.0 & 12.244996 & 11.820537 & 9.360162 \\
8.0 & 15.994992 & 15.283113 & 11.527638 \\
\hline
\end{tabular}

From these tables it is observed that the variation of $k_{0} h$ with $N^{2} h / g$ is to some extent insignificant compared to the variation with $\epsilon / h$ for fixed values of other parameters. Thus it appears that an exponentially stratified liquid with small Burnt-Vaisala parameter bounded by an inertial surface does not affect the wave motion set up by initial disturbances at the inertial surface significantly.

To visualize the nature of the wave motion set up, the form of $\tilde{\eta}(\tilde{x}, \tilde{t})$, obtained from (4.6) (due to an initial depression concentrated at the origin) is plotted in Figure 5.1 against $\tilde{x}$ between 1 and 6 with fixed $\tilde{t}=8$ and in Figure 5.2 against $\tilde{t}$ between 2 and 8 with fixed $\tilde{x}=1$ for the following four cases:

(i) $\epsilon / h=0.01, N^{2} h / g=0.1$

(ii) $\epsilon / h=0.01, N^{2} h / g=0$

(iii) $\epsilon / h=0, N^{2} h / g=0.1$

(iv) $\epsilon / h=0, N^{2} h / g=0$.

Similarly, $\left(\rho_{0} g^{1 / 2} l^{5 / 2} / A\right) \tilde{\eta}(\tilde{x}, \tilde{t})\left(=\tilde{\eta}^{*}\right.$, say) obtained from (4.16) (due to initial disturbance in the form of an impulse concentrated at the origin) is plotted against $\tilde{x}$ for fixed $\tilde{t}$ in Figure 5.3 and against $\tilde{t}$ for fixed $\tilde{x}$ in Figure 5.4. Figures 5.1 and 5.3 depict the wave profile at a particular instant. As the distance increases, the amplitude of the wave profiles asymptotically becomes zero, which is plausible since the initial disturbance is concentrated at the origin, and they die out at large distances. Figures 5.2 and 5.4 show the variation of $\tilde{\eta}$ at a particular place with time. As $\tilde{t}$ increases, the amplitudes are seen to be increasing which is rather unrealistic and arises due to strong singularity at the origin. Thus the qualitative feature of the wave motion at the inertial surface of stratified fluid are almost similar to those of the wave motion at the free surface of a homogeneous fluid.

Now in the figures, I and III correspond to a weakly stratified fluid with an inertial surface or a free surface while II and IV correspond to a homogeneous fluid with an inertial surface or a free surface. It is observed that in all the figures, the curves I and II are almost similar and similarly for III and IV. Thus weak stratification does not affect the wave motion significantly. However, the figures demonstrate that the presence of floating materials on the surface affects the wave motion significantly.

We may note that we have confined our study only on surface waves. So weak stratification does not affect the wave motion much. However, this affects the internal waves, which however, has not been studied here. 


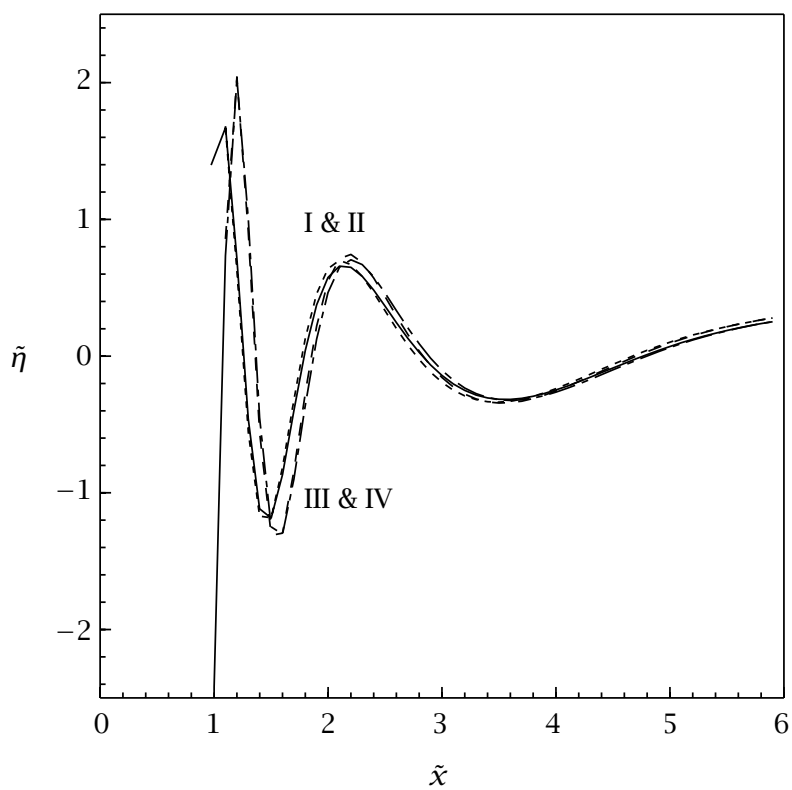

FIGURE 5.1. Wave profiles due to an initial disturbance in the form of an initial depression $(\tilde{t}=8.0), \epsilon / h=0.01, N^{2} h / g=0.1 \mathrm{I} ; \epsilon / h=0.01, N^{2} h / g=$ 0.0 II; $\epsilon / h=0.0, N^{2} h / g=0.1$ III; $\epsilon / h=0.0, N^{2} h / g=0.0$ IV.

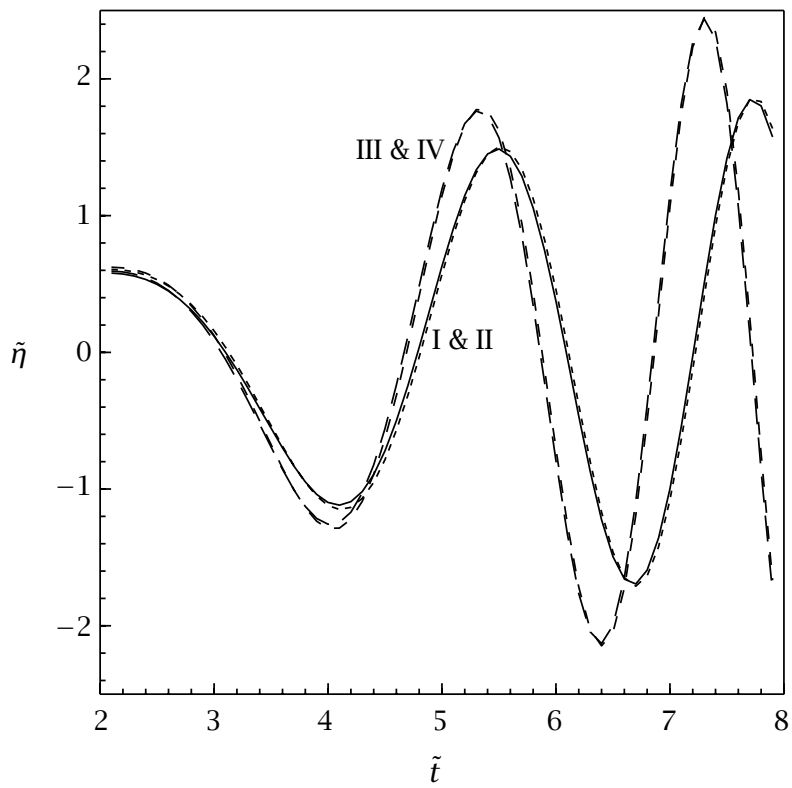

FIGURE 5.2. Wave profiles due to an initial disturbance in the form of an initial depression $(\tilde{x}=1.0), \epsilon / h=0.01, N^{2} h / g=0.1 \mathrm{I} ; \epsilon / h=0.01, N^{2} h / g=$ 0.0 II; $\epsilon / h=0.0, N^{2} h / g=0.1$ III; $\epsilon / h=0.0, N^{2} h / g=0.0$ IV. 


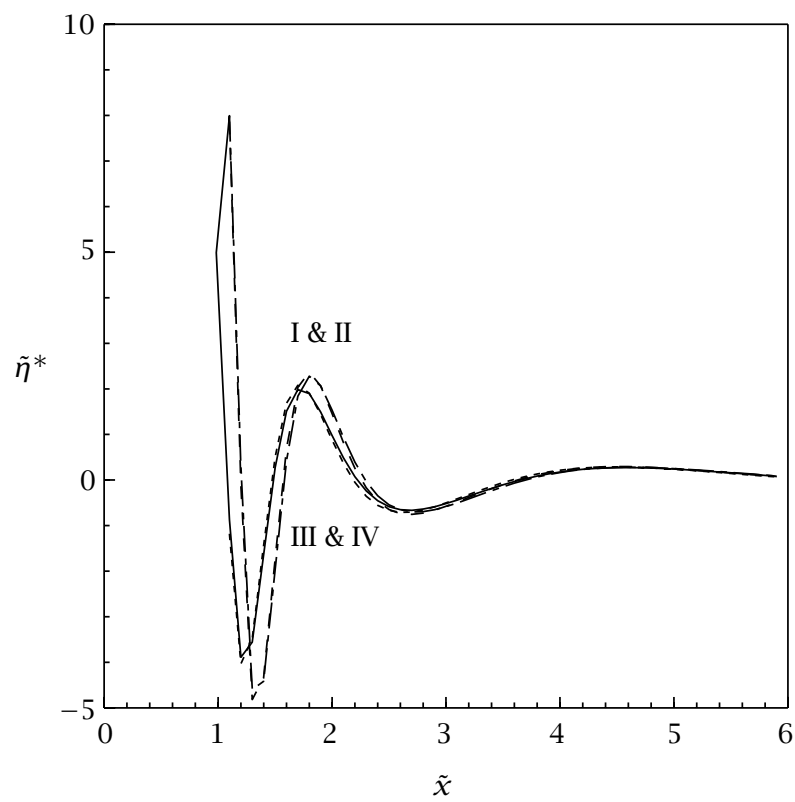

FIGURE 5.3. Wave profiles due to an initial disturbance in the form of an impulse $(\tilde{t}=8.0), \epsilon / h=0.01, N^{2} h / g=0.1 \mathrm{I} ; \epsilon / h=0.01, N^{2} h / g=0.0 \mathrm{II}$; $\epsilon / h=0.0, N^{2} h / g=0.1$ III; $\epsilon / h=0.0, N^{2} h / g=0.0$ IV.

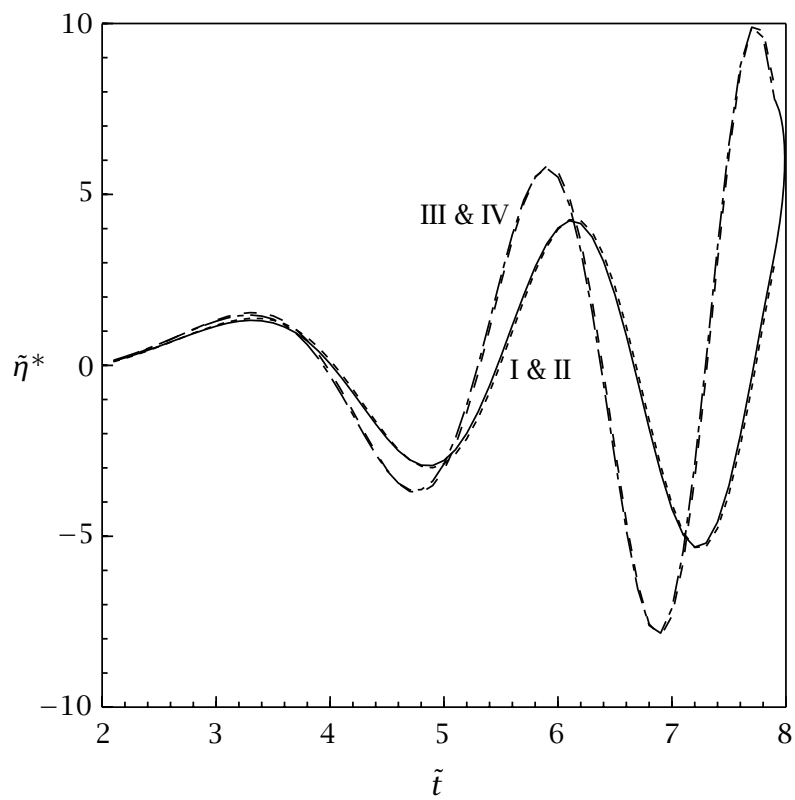

FIGURE 5.4. Wave profiles due to an initial disturbance in the form of an impulse $(\tilde{x}=1.0), \epsilon / h=0.01, N^{2} h / g=0.1 \mathrm{I} ; \epsilon / h=0.01, N^{2} h / g=0.0 \mathrm{II}$; $\epsilon / h=0.0, N^{2} h / g=0.1$ III; $\epsilon / h=0.0, N^{2} h / g=0.0$ IV. 
Acknowledgements. The authors thank Professor L. Debnath, Senior Fulbright Fellow, for some suggestions and going through an earlier draft of the paper during his visit to I.S.I., Calcutta. This work is partially supported by University Grants Commission, New Delhi.

\section{REFERENCES}

[1] K. Chaudhuri, Waves in shallow water due to arbitrary surface disturbances, Appl. Sci. Res. 19 (1968), 274-284. Zbl 159.59403.

[2] L. Debnath and U. B. Guha, The Cauchy-Poisson problem in an inviscid stratified liquid, Appl. Math. Lett. 2 (1989), no. 4, 337-340. MR 90i:76148. Zbl 703.76093.

[3] H. C. Kranzer and J. B. Keller, Water waves produced by explosions, J. Appl. Phys. 30 (1959), 398-407. MR 21\#1071. Zbl 085.20402.

[4] H. Lamb, Hydrodynamics, Dover, New York, 1945.

[5] B. N. Mandal, Water waves generated by disturbance at an inertial surface, Appl. Sci. Res. 45 (1988), no. 1, 67-73. Zbl 652.76011.

[6] B. N. Mandal and N. K. Ghosh, Waves generated by disturbances at an inertial surface in an ocean of finite depth, Proc. Indian Nat. Sci. Acad. Part A 55 (1989), no. 6, 906-911. CMP 1044091.

[7] _ Generation of water waves due to an arbitrary periodic surface pressure at an inertial surface in an ocean of finite depth, Proc. Indian Nat. Sci. Acad. Part A 56 (1990), no. 6, 521-531. MR 92a:86002. Zbl 714.76022.

[8] B. N. Mandal and S. Mukherjee, Water waves generated at an inertial surface by an axisymmetric initial surface disturbance, Int. J. Math. Educ. Sci. Technol. 20 (1989), no. 5, 743-747. Zbl 675.76015.

[9] J. J. Stoker, Water Waves: The Mathematical Theory with Applications, Interscience Publishers, Inc., New York, 1957. MR 21\#2438. Zbl 078.40805.

[10] S. L. Wen, A note on water waves created by surface disturbances, Int. J. Math. Educ. Sci. Technol. 13 (1982), 55-58. Zbl 482.76025.

Prity GHosh: Physics AND ApPlied MATHematics Unit, INDian STATISTICAL INSTITUTE, 203 B. T. ROAD, CALCUTTA 700 035, INDIA

Uma Basu: Department of Applied Mathematics, University of CAlcutta, 92 A. P. C. ROAD, CALCUTTA 700 009, INDIA

B. N. Mandal: Physics and ApPlied Mathematics Unit, Indian Statistical Institute, 203 B. T. ROAD, CALCUTTA 700 035, INDIA 


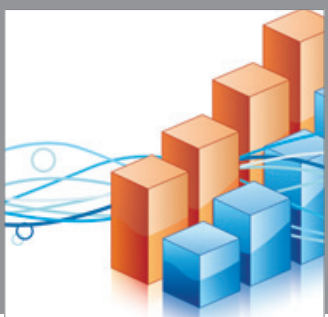

Advances in

Operations Research

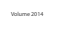

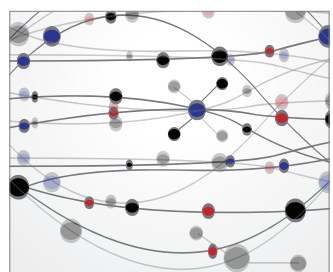

\section{The Scientific} World Journal
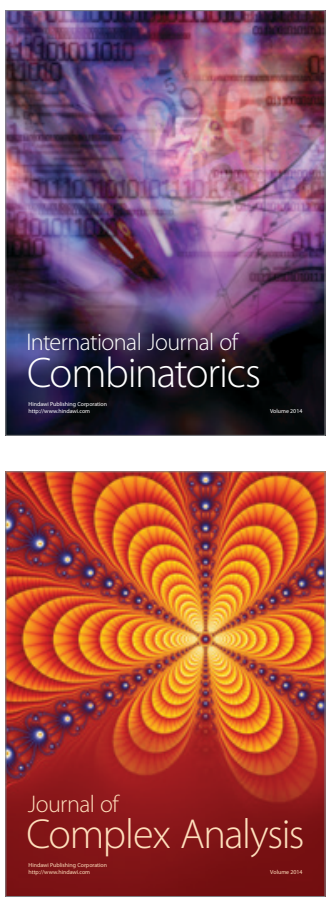

International Journal of

Mathematics and

Mathematical

Sciences
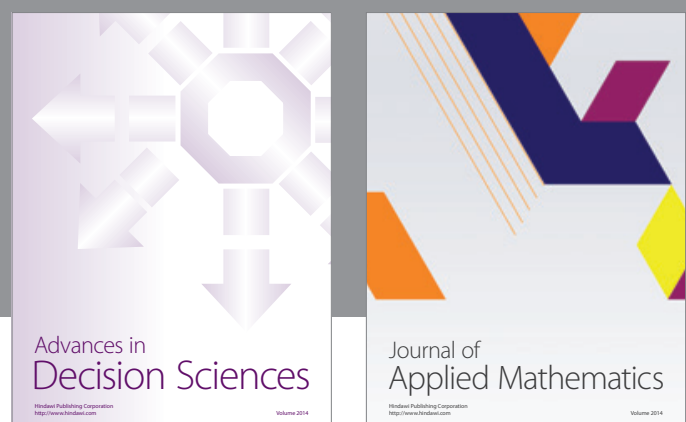

Journal of

Applied Mathematics
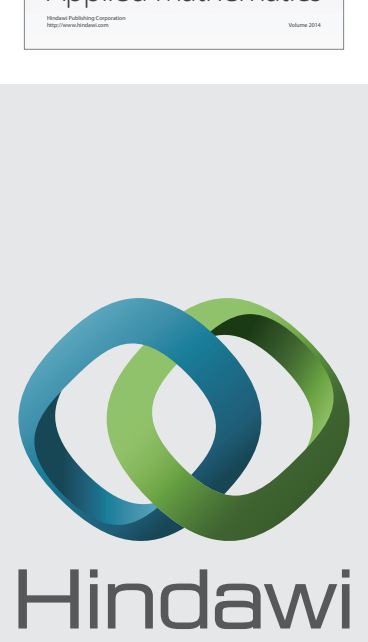

Submit your manuscripts at http://www.hindawi.com
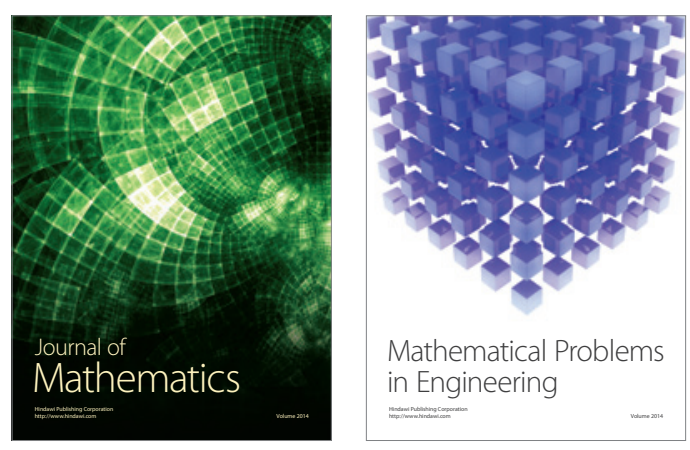

Mathematical Problems in Engineering
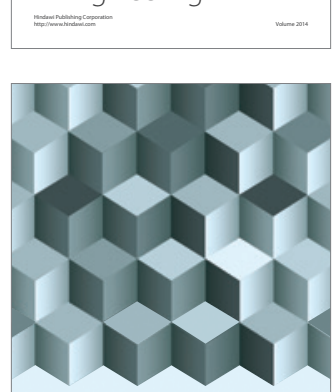

Journal of

Function Spaces
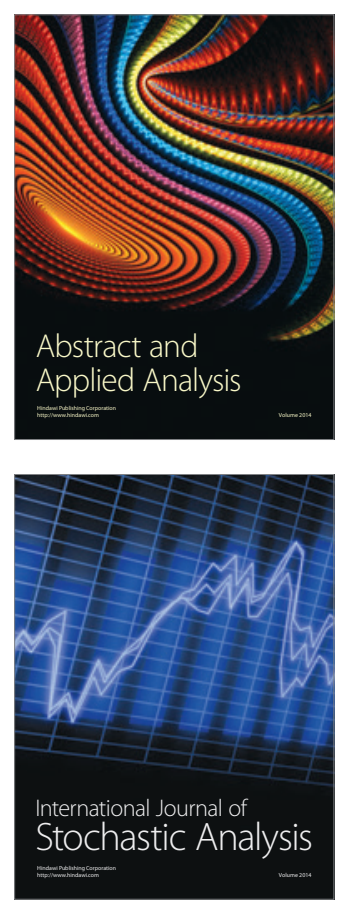

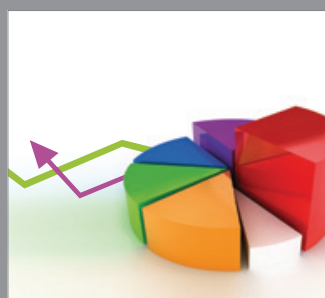

ournal of

Probability and Statistics

Promensencen
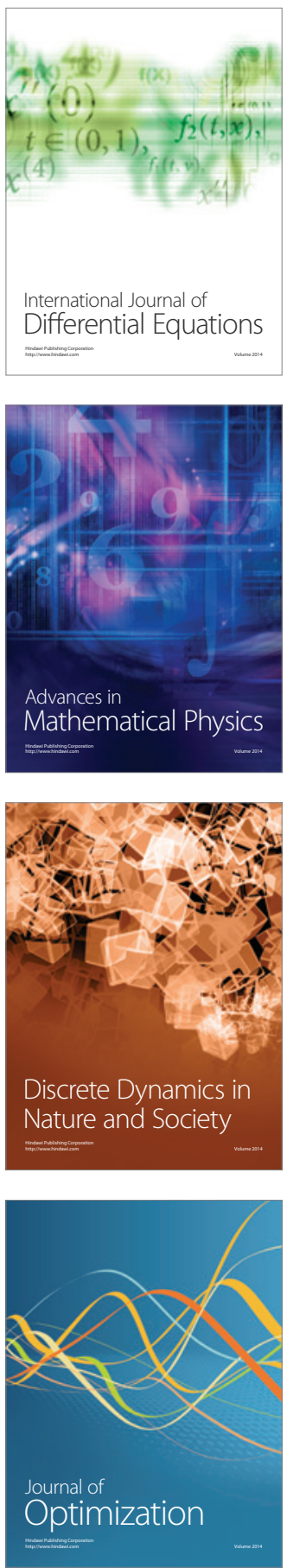